\title{
Female mating behaviour, sexual selection and chromosome I inversion karyotype in the seaweed fly, Coelopa frigida
}

\author{
ANDRÉ S. GILBURN* \& THOMAS H. DAY \\ Department of Genetics, University of Nottingham, Queens Medical Centre, Nottingham NG7 2UH, U.K.
}

\begin{abstract}
Previous studies of the seaweed fly, Coelopa frigida, have revealed the operation of several different forces of sexual selection. The overall pattern of mate choice seen in natural populations is not consistent with the predictions of indirect sexual selection as females do not express preferences that maximize the fitness of their offspring, even though the benefits from such choice are relatively large in this species. Thus, the maintenance of female mate choice for large male size must instead either be a result of a direct benefit to the female of mating with large males, or a side-effect of the evolution of another character, in other words pleiotropy. In order to separate these two alternatives the genetical basis of female mating behaviour needs to be studied. Previous studies have revealed associations between chromosomal inversion karyotype and both general female willingness to mate and mate choice for large male size, however these associations were lost after several generations of laboratory culture. Here several isokaryotypic lines from wild collections of flies were derived. The willingness to mate and mate choice of females from each line were determined. Pairs of lines of opposite inversion karyotype that significantly differed in either or both willingness to mate and mate choice were crossed. The mating behaviour and inversion karyotype of the $F_{2}$ progeny (all $F_{1}$ progeny are heterokaryotypes) were determined. Clear differences in the general levels of female willingness to mate were found between the two inversion homokaryotypes in several families, whereas variation in the strength of female choice for large male size were not revealed. It is suggested that mate choice in this species occurs as a pleiotropic effect of selection acting on female willingness to mate.
\end{abstract}

Keywords: chromosomal inversions, Coelopa frigida, female choice, sexual selection.

\section{Introduction}

In order to understand fully the mechanisms behind the evolution of female mate choice it is important to determine its genetic basis. If there is additive genetic variation in mate choice then indirect mechanisms of sexual selection can operate. Several recent studies have revealed that additive genetic variation does appear to be present in many female mate preferences (reviewed by Bakker \& Pomiankowski, 1995), suggesting that indirect sexual selection could be operating in many different species. The lack of such variation would prevent the operation of indirect sexual selection, but is consistent with the hypothesis that mate choice has evolved through direct benefits to the female or as a side-effect of genes that have primarily evolved through natural

*Correspondence and present address: School of Biological Sciences, University of Manchester, 3614 Stopford Building, Oxford Road, Manchester M13 9PT. E-mail: a.s.gilburn@man.ac.uk selection. Such a phenotype could arise through biases either in a female's sensory system (Ryan \& Rand, 1990; Ryan et al., 1990) or in the expression of female rejection responses that have initially evolved to reduce general willingness to mate (Rowe et al., 1994).

The mating system of the seaweed fly, Coelopa frigida, is characterized by a premating struggle during which females attempt to remove males forcibly. Female mate choice favours large male size in all natural populations (Gilburn et al., 1992, 1993, 1996; Gilburn \& Day, 1994a; Crean, 1997; Day \& Gilburn, 1997). Most of the genetic and much of the total variance in male size (Butlin et al., 1982; Gilburn \& Day, 1994b; Wilcockson et al., 1995; Day et al., 1996) is determined by a large chromosomal inversion system, with the $\alpha \alpha$ males being the largest, the $\beta \beta$ males the smallest and the heterokaryotype males being intermediate in size. Inversion karyotype is also a major determinant of viability, with the heterokaryotypes having considerably higher viability than either of the homokaryotypes in both laboratory and natural 
populations (Butlin et al., 1984; Butlin \& Day, 1989; Gilburn et al., 1996). Therefore mate choice on the basis of male size will have a correlated effect on inversion karyotype and, consequently, also on offspring fitness. In order to maximize offspring fitness, females should mate nonrandomly with respect to inversion karyotype (Gilburn \& Day, 1996). The $\beta \beta$ females are predicted to mate with large males in order to produce highly fit heterokaryotype offspring, and this is indeed what is observed in all natural populations so far studied (Day $\&$ Gilburn, 1997). The $\alpha \alpha$ females, in contrast, are predicted to mate with small males in order to increase the fitness of their offspring. However, in most populations the $\alpha \alpha$ females mate preferentially with large males (Day \& Gilburn, 1997).

If homokaryotype females did express preferences for mates of opposite homokaryotype in order to produce highly fit offspring, then female mate preferences would be expected to be inherited with the inversion system. An earlier study of the inheritance of female mating behaviour found that both female choice for large male size (and thus also karyotype) and general female willingness to mate appeared to be associated with the inversion system (Gilburn \& Day, 1994c). Female willingness to mate was determined from the acceptance rate of females within a particular karyotype. Female mate choice on the basis of male size was estimated from the logistic regression coefficient of female acceptance rate on male size for each karyotype. Comparisons of the acceptance rates of different karyotypes were made using a logistic analysis of variance model. The model was extended to include male size and an interaction term between karyotype and size (effectively an analysis of covariance). The latter term compares the strength of female mate choice on the basis of large male size for different karyotypes. In this study female willingness to mate and mate choice cosegregated with inversion karyotype when tested shortly after culture. However, when the line was retested after seven generations of laboratory culture, neither behaviour was significantly associated with karyotype. Consequently, it was not clear whether female mating behaviour was genuinely associated with inversion karyotype. The initial association could have been the result of genetic correlations between matingpreference genes and the inversion system, which had started to breakdown by the seventh generation. It was also noted that the association between general willingness to mate and karyotype appeared to be stronger than the association between mate choice for large male size and karyotype in the first generation females, and was also closer to significance and in the predicted direction within the seventh generation. It was possible that differences in preference for large male size seen in the first generation were a correlated response of differences in general willingness to mate. Female mate choice is mediated through a rejection response which obviously also determines general female willingness to mate. In addition, consistent differences are found between the willingness to mate of karyotypes across all populations, whereas considerable variation in female mating preferences have been observed both within and between populations (Crean, 1997).

The aim of the current study was to determine the mating behaviour exhibited by the homokaryotype females in the $F_{2}$ generation of families derived from pairs of isokaryotypic lines which significantly differ in either or both their willingness to mate or preference with respect to male size, and thus to establish which, if either, of these characters is the more clearly determined by inversion karyotype.

\section{Methods}

\section{Establishment of isokaryotypic lines}

Adults, collected from the wild as larvae in Northumberland, were allowed to eclose in the laboratory. Pairs of virgins were then placed in small pots containing fresh minced seaweed until larvae were observed. The adults were removed and their $A d h$ genotypes were determined. The $A d h$ locus can be used as a marker for the inversion system as the two most common alleles, $A d h-B$ and $A d h-D$, are found exclusively associated with the $\alpha$ and $\beta$ forms of the inversion, respectively (Day et al., 1982). The larvae from $\mathrm{BB} \times \mathrm{BD}$ or $\mathrm{BD} \times \mathrm{DD}$ crosses were transferred to larger pots of seaweed and allowed to develop. The larvae of all other pairs were discarded. The $F_{1}$ offspring from each pair were collected as virgins and paired in the same manner as their parents. The Adh genotypes of the live $\mathrm{F}_{1}$ adults that produced offspring were then also determined. The offspring of any $\mathrm{BD} \times \mathrm{BD}$ pairs were transferred to larger pots and allowed to develop. The $F_{2}$ offspring were then also paired and their $A d h$ genotypes were determined. The offspring of any pair of like homokaryotypes of the required type in the original parental cross were then used as the first generation of isokaryotypic lines. As there is no recombination in the region of the inversion in heterokaryotypes (Day et al., 1982), flies from these lines will carry two identical copies of the same inversion haplotype. The whole crossing scheme is outlined in Fig. 1.

\section{Determination of female mating behaviour in each line}

Once excess adults were available for an isokaryotypic line, virgin females were collected and placed in pots 


\begin{tabular}{|c|c|}
\hline Wild parents & $B B \times B D$ \\
\hline & 1 \\
\hline $\mathrm{F}_{1}$ & $\mathrm{BD} \times \mathrm{BD}$ \\
\hline & 1 \\
\hline $\mathrm{F}_{2}$ & $\mathrm{DD} \times \mathrm{DD}$ \\
\hline
\end{tabular}

Fig. 1 The establishment of a line homokaryotypic for the $\beta$ form of the chromosome I inversion system. Virgin adults collected from the wild as larvae were paired. The offspring of all $\mathrm{BB} \times \mathrm{DD}$ and $\mathrm{BD} \times \mathrm{DD}$ pairs were allowed to develop as larvae. Virgin adults from these pairs were crossed in the $F_{1}$ generation. Only larvae from $\mathrm{BD} \times \mathrm{BD}$ pairs were allowed to develop. Virgin adults from the $\mathrm{F}_{2}$ generation were then paired. The offspring from any $\mathrm{BB} \times \mathrm{BB}$ pairs (from $\mathrm{BD} \times \mathrm{DD}$ original crosses) and $\mathrm{DD} \times \mathrm{DD}$ crosses (from $\mathrm{BB} \times \mathrm{BD}$ original crosses) were used to establish $\alpha$ and $\beta$ homokaryotypic lines, respectively.

with cellulose wadding soaked with $0.5 \%$ mannitol solution and transferred in groups of up to 30 at $26^{\circ} \mathrm{C}$ for a two-day sex deprivation period. Virgin males from the laboratory population established from the original collection were also transferred to $26^{\circ} \mathrm{C}$ for a two-day sex deprivation period, but were placed individually in small pots containing minced seaweed. This period usually results in the males showing a very high mating vigour when they next encounter a female. Pairs of adults were then placed together in mating chambers and the result of the first mount attempted by a male was scored as either an acceptance, if the male succeeded in copulating with the female, or a rejection, if the female performed a rejection response and successfully prevented the male from copulating with her. The size of each male was determined by measuring wing length which is known to be highly correlated with other body dimensions (Butlin et al., 1982).

Willingness to mate was determined from the percentage of females of each group that accepted. The strength of female choice on the basis of male size was determined by logistic regression of female acceptance on male size using the Generalized Linear Interactive Modelling system (GLIM, Numerical Algorithm Group) with the logistic regression coefficient being used as a direct estimate of the strength of choice. In total 45 separate isokaryotypic lines were tested.

\section{The crosses}

Three pairs of lines of opposite homokaryotype were chosen in which the females of one line had a signifi- cantly higher willingness to mate than the females of the other. In all three pairs of lines the females did not differ in their strength of choice. Two further pairs of lines of opposite homokaryotype were also chosen which clearly differed in strength of choice but did not differ in acceptance rate. A further two pairs of lines were chosen which were found to differ in both acceptance rate and choice.

Virgin females from one line in each pair were placed together with males from the other line in a large sealed plastic tank containing minced seaweed (Fig. 2). The $F_{1}$ offspring, all of whom would be heterokaryotypes, were collected as adults and transferred to a fresh tank of minced seaweed. Virgin $F_{2}$ offspring were collected from the tank and stored at $4{ }^{\circ} \mathrm{C}$ until required. The acceptance rate and mate choice were calculated exactly as described above. The Adh genotype of all $\mathrm{F}_{2}$ females used in choice tests was determined. Logistic regression was performed to determine whether karyotype was associated with willingness to mate or female mate choice.

\section{Results}

The association of acceptance rate with karyotype

Three pairs of lines of opposite homokaryotype were chosen each of which had significantly different levels of willingness to mate, yet did not exhibit significantly different mating preferences. The $\alpha$-isokaryotypic line had the higher acceptance rate in two pairs $(\alpha 1$ and $\beta 1$; $\alpha 2$ and $\beta 2$ ), and the $\beta$-isokaryotypic line had the higher acceptance rate in the third pair $(\alpha 3$ and $\beta 3)$. The acceptance rates and preferences of the original lines and those of the $F_{2}$ offspring are shown in Table 1. In the first two pairs of lines willingness to mate was significantly associated with inversion haplotype in the $F_{2}$ offspring ( $\alpha 1$ vs. $\beta 1, F_{1,119}=23.99, P<0.001 ; \alpha 2$ vs. $\left.\beta 2, F_{1,92}=13.69, P<0.001\right)$ and in both cases the $\alpha$ -

$\begin{array}{cc}\text { Isokaryotypic lines } & \mathrm{BB} \times \mathrm{DD} \\ \mathrm{F}_{1} & \mathrm{BD} \times \mathrm{BD} \\ & 1\end{array}$

$\mathrm{F}_{2}$ females used in experiments $\quad \mathrm{BB}, \mathrm{BD} \& \mathrm{DD}$

Fig. 2 The crossing scheme used to establish $\mathrm{F}_{2}$ offspring from pairs of homokaryotypic lines of opposite chromosome 1 inversion karyotype. 
Table 1 The acceptance rates and preferences (determined by logistic regression analysis of female acceptance rate on male size) of the original isokaryotypic lines used in the crosses, together with those of the isokaryotypic $\mathrm{F}_{2}$ offspring of the crosses between each pair of lines. Acceptance rates are expressed as proportion of males accepted. Pairs of figures in bold are significantly different

\begin{tabular}{lccccc}
\hline & \multicolumn{2}{c}{ Pure line } & & \multicolumn{2}{c}{$F_{2}$ offspring } \\
\cline { 2 - 3 } \cline { 5 - 6 } Line & $\begin{array}{c}\text { Acceptance } \\
\text { rate }\end{array}$ & Preference & & $\begin{array}{c}\text { Acceptance } \\
\text { rate }\end{array}$ & Preference \\
\hline$\alpha 1$ & $\mathbf{0 . 7 8}$ & 1.30 & & $\mathbf{0 . 8 8}$ & 0.89 \\
$\beta 1$ & $\mathbf{0 . 1 7}$ & 1.42 & & $\mathbf{0 . 4 8}$ & 0.45 \\
$\alpha 2$ & $\mathbf{0 . 7 1}$ & 0.43 & & $\mathbf{0 . 6 8}$ & 0.58 \\
$\beta 2$ & $\mathbf{0 . 2 6}$ & 0.71 & & $\mathbf{0 . 2 7}$ & 0.22 \\
$\alpha 3$ & $\mathbf{0 . 3 8}$ & 0.64 & & 0.36 & -0.52 \\
$\beta 3$ & $\mathbf{0 . 7 9}$ & 0.35 & & 0.30 & -0.03 \\
$\alpha 4$ & 0.39 & $-\mathbf{0 . 2 2}$ & & 0.89 & 1.12 \\
$\beta 4$ & 0.56 & $\mathbf{1 . 4 2}$ & & 0.80 & 1.29 \\
$\alpha 5$ & 0.50 & $\mathbf{- 0 . 1 8}$ & & 0.63 & 0.76 \\
$\beta 5$ & 0.45 & $\mathbf{0 . 7 1}$ & & 0.72 & 1.51 \\
$\alpha 6$ & $\mathbf{0 . 8 6}$ & $\mathbf{1 . 3 8}$ & & $\mathbf{0 . 7 5}$ & 1.11 \\
$\beta 6$ & $\mathbf{0 . 2 7}$ & $\mathbf{0 . 3 0}$ & & $\mathbf{0 . 4 7}$ & 0.66 \\
$\alpha 7$ & $\mathbf{0 . 3 8}$ & $\mathbf{0 . 5 8}$ & $\mathbf{0 . 5 3}$ & 1.33 \\
$\beta 4$ & $\mathbf{0 . 5 6}$ & $\mathbf{1 . 4 2}$ & $\mathbf{0 . 3 2}$ & 0.76 \\
\hline & & & &
\end{tabular}

isokaryotypes were more willing to mate. There was no difference in mate choice between the isokaryotypes in the $\mathrm{F}_{2}$ of these two pairs of lines $(\alpha 1$ vs. $\beta 1$, $F_{1,118}=0.65, \quad P=0.42 ; \alpha 2$ vs. $\beta 2, \quad F_{1,91}=0.46$, $P=0.50)$. There was no difference in either acceptance rate ( $\alpha 3$ vs. $\beta 3, F_{1,73}=0.08, P=0.78$ ) or mate choice ( $\alpha 3$ vs. $\left.\beta 3, F_{1,72}=0.29, P=0.59\right)$ in the isokaryotypic $\mathrm{F}_{2}$ offspring from the pair of lines where the $\beta$ isokaryotypes originally had the higher acceptance rate.

\section{The association of mate choice with karyotype}

Two pairs of lines ( $\alpha 4$ and $\beta 4 ; \alpha 5$ and $\beta 5$ ) were chosen which had significantly different preferences but similar acceptance rates. In both pairs the original $\beta$-isokaryotypic line exhibited a strong choice for large male size and the $\alpha$-isokaryotypic line exhibited no preference (Table 1). There were no significant differences found in either mate choice ( $\alpha 4$ vs. $\beta 4, F_{1,77}=0.05$, $P=0.82 ; \alpha 5$ vs. $\left.\beta 5, \quad F_{1,49}=0.50, P=0.61\right)$ or acceptance rate $\left(\alpha 4\right.$ vs. $\beta 4, F_{1,78}=2.08, P=0.15 ; \alpha 5$ vs. $\left.\beta 5, F_{1,50}=0.92, P=0.34\right)$ in the isokaryotypic $\mathrm{F}_{2}$ offspring from either pair of lines.

\section{The association of acceptance rate and mate choice with karyotype}

Two pairs of lines were chosen which had significantly different levels of willingness to mate and significantly different mating preferences. In the first pair $(\alpha 6$ and $\beta 6)$ the $\alpha$-isokaryotypic line had the higher acceptance rate and the stronger preference, whereas in the second pair $(\alpha 7$ and $\beta 4)$ the $\beta$-isokaryotypic line had the higher acceptance rate and the stronger preference. There was a significant difference in willingness to mate between the $\mathrm{F}_{2}$ isokaryotypes ( $\alpha 6$ vs. $\beta 6, F_{1,71}=7.36, P=0.008$; $\alpha 7$ vs. $\left.\beta 4, F_{1,83}=4.44, P=0.038\right)$ in both pairs, with the $\alpha$-isokaryotypes having the higher willingness to mate in both cases. There was no difference in mate choice between the isokaryotypes in either of these two pairs of lines ( $\alpha 6$ vs. $\beta 6, F_{1,70}=0.30, P=0.58 ; \alpha 2$ vs. $\left.\beta 2, F_{1,82}=1.12, P=0.29\right)$.

\section{Discussion}

In the three crosses in which the $\alpha$-isokaryotypic line had the higher willingness to mate, this appears to have been inherited with the $\alpha$-form of the inversion through to the $\mathrm{F}_{2}$ offspring. This result is in agreement with the previous study on the inheritance of female mating behaviour (Gilburn \& Day, 1994c) and is consistent with the survey of mating behaviour in wild-collected females, in which the $\alpha$-homokaryotypes were found to be more willing to mate than either the $\beta$-homokaryotypes or the heterokaryotypes in all 16 populations studied (Gilburn et al., 1992, 1993, 1996; Gilburn \& Day, 1994a; Crean, 1997; Day \& Gilburn, 1997). There was also a significant difference in the acceptance rate of the $F_{2}$ offspring in one of the pairs of lines in which the $\beta$-line was originally associated with higher willingness to mate. However, in this case, the acceptance rates appeared to have become reversed in the $F_{2}$, with the $\alpha$-homokaryotype females having a higher willingness to mate. This suggests that the $\alpha$-form of the inversion system is generally associated with higher willingness to mate and that variation in environmental conditions was probably responsible for the original difference between the two lines.

There was no evidence in any of the crosses of mate choice with respect to male size showing a clear pattern of association with inversion karyotype. This result seems to conflict with the earlier study of the inheritance of female mating preferences in C. frigida, which apparently showed a clear difference in the choice between female $F_{1}$ offspring of different karyotype (Gilburn \& Day, 1994c), and other studies, which have found an apparent association between choice and karyotype (Gilburn et al., 1993, 1996; 
Gilburn \& Day, 1994a). One possible explanation is that there is a genetic difference in mating preference between karyotypes, but this difference occurs as a pleiotropic effect of genes determining the general willingness to mate. Consequently, stronger and more consistent associations will be found between willingness to mate and inversion karyotype, than between mate choice and inversion karyotype. The female rejection response that generates the premating struggle may have evolved in order to reduce the general frequency of copulation as a result of costs associated with mating, rather than as a method of rejecting specific unpreferred mates. Variation in the efficiency of rejecting males of different size could then lead to the evolution of an apparent preference for large male size, as small males are likely to be easier and more cost-efficient to reject than large males. A recent study of another seaweed fly, Coelopa ursina, has revealed that the large-male mating advantage in this species occurs as a direct result of larger males being able to survive female rejection responses for longer than small males (Crean \& Gilburn, 1998). A similar study in $C$. frigida has also revealed a positive association between the length of premating struggles and both male size and mating success (C. Wilson \& A.S. Gilburn, unpubl. results).

The association between inversion karyotype and male size would mean that females are effectively expressing a preference for $\alpha$-homokaryotype males even if the choice occurs as a side-effect. As a result of this preference the $\beta$-homokaryotype females would tend to overproduce highly fit heterokaryotypic offspring. However, the $\alpha$-homokaryotype females would be increasing their production of unfit homokaryotype offspring. Any allele increasing the willingness to mate of $\alpha$-homokaryotype females may well then be selected if it also had a correlated effect on mate choice and reduced the preference for large male size. This could provide an explanation for the consistently higher acceptance rates exhibited by $\alpha$-homokaryotype females over $\beta$-homokaryotype females in all populations (Gilburn et al., 1992, 1993, 1996; Gilburn \& Day, 1994a; Crean, 1997; Day \& Gilburn, 1997). Alternatively, the cost of performing a mate rejection response may vary between females of different karyotypes, perhaps as a result of differences in the epistatic interactions between genes affecting willingness to mate and alleles fixed on one form of the inversion system or another.

Thus, it is concluded that all the genetic variation in mate choice associated with the inversion system could be the result of pleiotropic effects of loci determining willingness to mate, which do clearly differ between inversion karyotypes. Even so, both Fisherian and good genes sexual selection have been found to operate in this species (Gilburn et al., 1993, 1996; Gilburn \& Day, 1994a), although perhaps only as a side-effect of genetic variation in female reluctance to mate. This clearly shows that multiple forces of sexual selection can be operating within a single species. Thus the discovery of a selective force maintaining mate choice within a species should not lead to the conclusion that this provides an adaptive explanation for mate choice. Only when the relative strengths of each selective force have been estimated can inferences about the evolution of mate choice be made. In $C$. frigida the pattern of preferences seen in natural populations is only consistent with direct sexual selection and pleiotropy. This study reveals apparent genetic variation in female willingness to mate but not in female mate choice. Thus it is concluded that the apparent mate choice for large male size occurs as a pleiotropic effect of selection acting on willingness to mate even though the potential benefits to good genes sexual selection are relatively large in this species. This adds empirical support to the predictions of Kirkpatrick \& Barton (1997) who suggested that forces of indirect sexual selection would be too weak to explain the evolution of female mate choice.

The seaweed fly mating system bears considerable resemblance to that of the gerrids. The gerrid mating system is characterized by sexual conflict between the sexes which results in females performing violent mate rejection responses. Larger males have been found to survive premating struggles more successfully than small males resulting in a large-male mating advantage (Fairbairn, 1988, 1990; Sih \& Krupa, 1992; Fairbairn \& Preziosi, 1994; Arnqvist et al., 1996). In other words, sexual selection occurs as a side-effect of selection acting to reduce female willingness to mate (Rowe et al., 1994). There is also considerable variation in the strength of sexual selection favouring male size within the gerrids (Rowe et al., 1994). This is believed to occur because of variation in a whole range of environmental variables like predation risk, male harassment and food availability. In order to understand fully why there is so much within- and between-population variation in the intensity of sexual selection in seaweed flies it is important to study the factors determining female willingness to mate. Environmental variation in factors that could affect female willingness to mate, like the availability of oviposition sites and food, the tidal cycle, operational sex ratio and temperature could result in complex patterns in the apparent strength and direction of mate preferences for male size. Consequently, investigations are currently being performed into the factors determining female willingness to mate in seaweed flies. 


\section{Acknowledgements}

We thank Caroline Crean for comments on an earlier version of the manuscript. This work was supported by grant no. GR3/8826(ML17) from the Natural Environment Research Council (to T.H.D.).

\section{References}

ARNQVIST, G., ROWE, L., KRUPA, J. AND SIH, A. 1996. Assortative mating by size: a meta-analysis of mating patterns in water striders. Evol. Ecol., 10, 265-284.

BAKKER, T. C. M. AND POMIANKOWSKI, A. 1995. The genetic basis of female mate preferences. J. Evol. Biol., 8, 129-171.

BUTLIN, R. K. AND DAY, T. H. 1989. Environmental correlates of inversion frequencies in natural populations of seaweed flies (Coelopa frigida). Heredity, 62, 223-232.

BUTLIN, R. K., READ, I. L. AND DAY, T. H. 1982. The effects of a chromosomal inversion on adult size and male mating success in the seaweed fly, Coelopa frigida. Heredity, 49, 51-62.

BUTLIN, R. K., COLlinS, P. M. AND DAY, T. H. 1984. The effect of larval density on an inversion polymorphism in the seaweed fly, Coelopa frigida. Heredity, 52, 415-423.

CREAN, C. S. 1997. Variation in Female Mate Preferences in the Seaweed Fly, Coelopa frigida. Ph.D. Thesis, University of Nottingham.

CREAN, C. S. AND GILBURN, A. S. 1998. Sexual selection as a sideeffect of sexual conflict in the seaweed fly, Coelopa ursina (Diptera: Coelopidae). Anim. Behav., 56, 1405-1410.

DAY, T. H. and GILBURN, A. S. 1997. Sexual selection in seaweed flies. Adv. Study Behav., 26, 1-58.

DAY, T. H., DOBSON, T., HILlIER, P.C., PARKIN, D. T. AND ClARKE, B. C. 1982. Associations of enzymic and chromosomal polymorphisms in the seaweed fly, Coelopa frigida. Heredity, 48, 35-44.

DAY, T. H., CREAN, C. S., GILBURN, A. S., SHUKER, D. M. AND WILCOCKSON, R. W. 1996. Sexual selection in seaweed flies: genetic variation in male size and its reliability as an indicator in natural populations. Proc. R. Soc. B., 263, 1127-1134.

FAIRBAIRN, D. J. 1988. Sexual selection for homogamy in the Gerridae: an extension of Ridley's comparative approach. Evolution, 42, 1212-1222.

FAIRBAIRN, D. J. 1990. Factors influencing sexual size dimorphism in temperate waterstriders. Am. Nat., 136, 61-86.

FAIRBAIRN, D. J. AND PREZIOSI, R. F. 1994. Sexual selection and the evolution of allometry for sexual size dimorphism in the waterstrider, Aquarius remigis. Am. Nat., 144, 101-108.
GILBURN, A. S. AND DAY, T. H. 1994a. Evolution of female choice in seaweed flies: Fisherian and good genes mechanisms operate in different populations. Proc. R. Soc. B., 255, 159-165.

GILBURN, A. S. AND DAY, T. H. 1994b. Sexual dimorphism, sexual selection and the $\alpha \beta$ inversion polymorphism in the seaweed fly, Coelopa frigida. Proc. R. Soc. B., 257, 303-309.

GILBURN, A. S. AND DAY, T. H. 1994c. The inheritance of female mating behaviour in the seaweed fly, Coelopa frigida. Genet. Res., 64, 19-25.

GILBURN, A. S. AND DAY, T. H. 1996. The evolution of female choice when the preference and the preferred trait are linked to the same inversion system. Heredity, 76, 19-27.

GILBURN, A. S., FOSTER, S. P. AND DAY, T. H. 1992. Female mating preference for large size in Coelopa frigida. Heredity, 69, 209-216.

GILBURN, A. S., FOSTER, S. P. AND DAY, T. H. 1993. Genetic correlation between a female mating preference and the male preferred character in the seaweed fly, Coelopa frigida. Evolution, 47, 1788-1795.

GILbURN, A. S., CREAN, C. S. AND DAY, T. H. 1996. Sexual selection in natural populations of seaweed flies: variation in the offspring fitness of females carrying different inversion karyotypes. Proc. R. Soc. B., 263, 249-256.

KIRKPATRICK, M. AND BARTON, N. H. 1997. The strength of indirect selection on female mating preferences. Proc. Natl. Acad. Sci. U.S.A., 94, 1282-1286.

Rowe, L., ARnQvist, G., SIH, A. AND KRUPA, J. J. 1994. Sexual conflict and the evolutionary ecology of mating patterns: water striders as a model system. Trends Ecol. Evol., 9, 289293.

RYAN, M. J. AND RAND, A. S. 1990. The sensory basis of sexual selection for complex calls in the tungara frog, Physalaemus pustulosus (sexual selection for sensory exploitation). Evolution, 44, 305-314.

RYAN, M. J., FOX, J. H., WILCYNSKI, W. AND RAND, A. S. 1990. Sexual selection for sensory exploitation in the frog Physalaemus pustulosus. Nature, 343, 66-67.

SIH, A. AND KRUPA, J. J. 1992. Predation risk, food deprivation and non-random mating by size in the stream-water strider, Aquarius remigis. Behav. Ecol. Sociobiol., 31, 51-56.

WILCOCKSON, R. W., CREAN, C. S. AND DAY, T. H. 1995. Heritability of a sexually selected character. Nature, 374, $158-159$. 